\title{
La « ligne droite » et la « ligne sinueuse »: reportage et littérature dans les Récits des marais rwandais de Jean Hatzfeld
}

"Straight Line" and "Winding Line": Reportage and Literature in Jean Hatzfeld's

Récits des marais rwandais

\section{Maud Lecacheur}

\section{OpenEdition \\ Journals}

Édition électronique

URL : https://journals.openedition.org/recherchestravaux/3683

DOI : 10.4000/recherchestravaux.3683

ISSN : 1969-6434

Éditeur

UGA Éditions/Université Grenoble Alpes

Édition imprimée

ISBN : 978-2-37747-301-4

ISSN : 0151-1874

Référence électronique

Maud Lecacheur, «La « ligne droite » et la « ligne sinueuse » : reportage et littérature dans les Récits des marais rwandais de Jean Hatzfeld », Recherches \& Travaux [En ligne], 98 | 2021, mis en ligne le 28 juin 2021, consulté le 09 août 2021. URL : http://journals.openedition.org/recherchestravaux/3683 DOI : https://doi.org/10.4000/recherchestravaux.3683

Ce document a été généré automatiquement le 9 août 2021

(c) Recherches \& Travaux 
La « ligne droite » et la « ligne sinueuse $»$ : reportage et littérature dans les Récits des marais rwandais de Jean Hatzfeld

\author{
"Straight Line" and "Winding Line": Reportage and Literature in Jean Hatzfeld's \\ Récits des marais rwandais
}

\title{
Maud Lecacheur
}

1 Grand reporter de guerre pour Libération à partir de 1975, Jean Hatzfeld expérimente, en se confrontant aux secousses du génocide tutsi au Rwanda, une littérature fondée sur la collecte de témoignages. Au cours des années 2000, les recueils qu'il publie au fil de ses séjours à Nyamata composent une trilogie rwandaise: Dans le nu de la vie rassemble en 2000 les récits de quatorze rescapés; Une saison de machettes recueille en 2003 les propos d'une bande de tueurs alors emprisonnés; La Stratégie des antilopes retrouve en 2007 les témoins des précédents volumes dans le contexte très tendu de la réconciliation, une fois les tueurs rentrés à Nyamata ${ }^{1}$. À ces textes sur le génocide des Tutsis s'ajoutent deux autres livres, Englebert des collines en 2014 et Un papa de sang en 2016, mais les trois premiers livres forment une unité éditoriale : republiés en un seul volume en 2014 sous le titre Récits des marais rwandais, ils ont paru dans la collection «Fiction \& Cie » au Seuil, tandis que les deux autres ont été publiés dans la collection «Blanche » de Gallimard.

2 La trilogie marque une seconde entrée de Jean Hatzfeld dans le champ littéraire : s'il était déjà l'auteur de plusieurs romans inspirés de son expérience de correspondant de guerre, c'est désormais comme auteur de non-fiction qu'il est identifié par le public, auprès duquel il rencontre un grand succès. La trilogie remporte en effet de nombreux prix : le prix France Culture, le prix Joseph Kessel, le prix Médicis et le prix Ryszard Kapuściński, récompenses qui accentuent encore l'impression d'une œuvre brouillant les frontières entre reportage et littérature. Soulignons qu'avec cette trilogie, la démarche de Jean Hatzfeld se rapproche du travail de Svetlana Alexievitch avec ses 
récits de voix de l'ex-Empire soviétique. Le parallèle entre les deux auteurs est d'autant plus séduisant qu'ils appartiennent à la même génération (nés dans l'immédiat aprèsguerre, ils sont fascinés par la Seconde Guerre mondiale et héritent de l'« ère du témoin $\left.{ }^{2} »\right)$, qu'ils ont tous deux eu une carrière de journaliste, et qu'ils ont développé une méthode semblable de collecte d'entretiens auprès des oubliés de l'histoire.

Pourtant, malgré l'évidente proximité de cette démarche avec le travail du reporter, Jean Hatzfeld ne cesse de revendiquer pour la trilogie une rupture forte avec l'expérience et l'écriture du journalisme: dans ses entretiens comme dans le discours réflexif de chaque livre, il s'agit moins pour lui de conférer une valeur littéraire au genre du reportage ou de situer son travail à la lisière du journalisme, que de formuler la conscience progressive des écarts entre reportage et littérature. Dans de nombreux entretiens, il présente sa rencontre avec le génocide comme une bifurcation radicale dans son parcours, par exemple en 2012 lors des Assises internationales du roman : « Le reportage est mon métier, il est formidable, il est aussi ma famille, mais je n'ai pas l'impression que mes livres relèvent du travail de journaliste, ni dans la démarche, ni dans la manière d'être, ni dans l'écriture, au contraire ${ }^{3}$.» La confrontation de Jean Hatzfeld au génocide coïncide ainsi avec le congé donné au journalisme, d'un point de vue matériel (il renouvelle annuellement, à partir de 1998, son congé sans solde auprès de Libération) et d'un point de vue méthodologique. Cette opposition est thématisée dans La Stratégie des antilopes :

Comment écrire la parole des autres ? [...] Ce n'est pas facile, ce peut être très complexe, mais c'est un travail d'écriture naturel, si la motivation est essentiellement littéraire, si on est mu par l'ambition d'entraîner le lecteur dans l'univers génocidaire, le désir de transmettre une histoire. [...] L'histoire, les gens, les rencontres, les phrases et les images se mêlent dans une écriture qui traduit différemment un événement. Cette littérature-là est plus sinueuse, plus lente et plus tardive, plus métaphorique, plus inspirée, mais elle est tout aussi efficace, pour acheminer l'information d'un point à un autre, lorsque la ligne droite, la voie directe, celle qu'empruntent en pionniers les journalistes, est barrée ; et elle l'est systématiquement après un génocide [...] parce que les journalistes et tout autant, sinon plus, leurs lecteurs ou téléspectateurs butent sur un événement aussi extraordinaire. (SA, p. 206-207)

Dans ce passage, journalisme et littérature s'opposent moins par leurs visées - ils travaillent également à informer le lecteur sur un événement, à transmettre au public une réalité à laquelle il n'a pas accès - que par leur méthode, leur cheminement respectif : l'approche détournée, oblique, qui serait propre à la littérature, permettrait de contourner l'impossibilité initiale rencontrée par le journalisme dans le désir d'accéder à la compréhension du génocide, en faisant le pari d'une autre temporalité. Cette citation éclaire la manière dont l'écrivain s'efforce d'inventer à mesure une autre méthode, qui passe par la fabrique d'un témoignage indirect : ne pouvant témoigner d'une réalité dont il n'a pas fait l'expérience directe, Jean Hatzfeld travaille à recueillir les témoignages d'autrui.

5 La distinction d'une ligne «droite " ou «directe » du journalisme et d'une ligne "sinueuse » de la littérature invite à interroger la négociation des rapports entre reportage et littérature dans la trilogie selon trois modalités : l'abandon du journalisme au profit d'une démarche littéraire, à travers une éthique de l'écoute et une poétique du témoignage indirect ; les enjeux de ces rapports du point de vue de la réception ; les liens entre politique et littérature dans la trilogie. 


\section{Du journalisme à la littérature : éthique de l'écoute et poétique du témoignage indirect}

6 Pour comprendre cette rupture, il faut rappeler le contexte dans lequel Jean Hatzfeld écrit puis publie Dans le nu de la vie à la toute fin des années 1990. Au-delà du désir de s'affranchir de ce qu'il appelle les "règles d'or » du journalisme, ce congé donné au reportage intervient à un moment de crise profonde de la presse écrite quotidienne nationale, qui touche de plein fouet Libération: en 1996, le journal perd son indépendance capitalistique ; entre 2000 et 2015, sa diffusion est divisée de moitié; en 2006, la rédaction, qui comprenait 350 salariés, n'en compte plus que $200^{4}$. Cette crise économique se traduit par une exigence croissante de brièveté des articles et la diminution des budgets permettant de financer de grands reportages: dans un entretien accordé à Jean Birnbaum en 2010, Jean Hatzfeld explique avoir trouvé dans la littérature un refuge, dans un contexte marqué par la marginalisation du grand reporter au sein des journaux à la fin des années $1980^{5}$. D'autre part, au moment où il entreprend d'écrire un livre sur le génocide des Tutsis, l'essor des mooks ${ }^{6}$ n'a pas encore eu lieu: il faut attendre la fin des années 2000 pour qu'apparaissent des revues accueillant des textes qui excèdent les normes du reportage ${ }^{7}$. Publiée de 2000 à 2007, la trilogie documentaire correspond ainsi à un moment de vacance dans le champ journalistique, avant l'invention de nouveaux modèles. C'est donc aussi contre l'absence d'espace pour le journalisme littéraire que s'écrivent les livres.

7 Un autre phénomène explique cet abandon progressif du journalisme, à savoir le constat de l'échec massif de la communauté médiatique internationale à rendre compte du génocide en 1994. Jean Hatzfeld ne cesse de répéter qu'à la suite de ses confrères, il s'est trompé d'objet, en se détournant du mutisme des rescapés pour transmettre les épisodes d'exode massif des Hutus vers le Zaïre. Dès Dans le nu de la vie, les rescapés apparaissent comme les oubliés de l'Histoire et les laissés-pour-compte du génocide. Le projet de collecte de voix à Nyamata se construit contre la couverture médiatique de l'événement et à contretemps de l'urgence de l'actualité. Dans les témoignages des rescapés, le journaliste incarne un contre-modèle pour le collecteur de voix, comme le suggère le récit d'Innocent Rwililiza: "Les reporters passaient devant la porte sans même frapper, parce qu'ils étaient trop occupés pour s'attarder devant des gens qui n'échangeaient plus rien » (DNV, p. 108). D'où la nécessité d'opérer un pas de côté vis-àvis de la posture du journaliste. Au moment où, à la fin des années 1990, le journalisme se définit de plus en plus par l'image et la vitesse, la méthode de Jean Hatzfeld se construit en réaction à ces deux phénomènes : au lieu de la vitesse, l'extrême lenteur, la temporalité de l'après-coup et non de l'actualité, puisque le premier livre paraît six ans après le génocide, et que l'écrivain consacre environ deux ans à chaque volume; au lieu de l'image, les voix, avec le choix quasi-exclusif de l'écoute, parti pris mis en avant dans le chapitre « Une précision en chemin » dans Le nu de la vie où s'explicite le protocole du livre: «Lors des premières discussions avec Sylvie Umubyeyi, puis avec Jeannette Ayinkamiye et d'autres personnes présentées par Sylvie, il m'apparait tout de suite évident de consacrer du temps à les écouter» (DNV, p.173). En choisissant d'enregistrer pendant des heures, à plusieurs reprises et sur plusieurs années, les mêmes personnes, Jean Hatzfeld s'inscrit ainsi dans cette tendance de l'art documentaire qu'a identifiée Aline Caillet, qui se traduit par un glissement de la visualité vers l'écoute ${ }^{8}$. La pratique de l'entretien se déplace de l'expérience de 
l'interview journalistique aux modèles hérités des sciences humaines et sociales, entre récit de vie et témoignage historique, ce dont attestent les références convoquées par l'écrivain, de Primo Levi à Christopher Browning en passant par Claude Lanzmann. La méthode de l'enquêteur de Shoah fait directement écho à cet absolu de l'écoute, comme en témoigne un texte d'hommage au réalisateur:

Aller jusqu'au bout, relever le défi ultime, filmer la mort sans images, c'est d'abord l'entendre. C'est donner naissance à cette parole, l'écouter, la recueillir pour la monter en résonance avec d'autres paroles, avec des voix et des visages, des rires, des larmes, donc des personnages [...]. Ce montage qui donne vie à la disparition a bouleversé des foules de spectateurs et perturbé des cinéastes. N’oublions pas que la parole l'a précédés.

8 L'hommage à Lanzmann, dont le cinéma documentaire a marqué selon Shoshana Felman l'avènement de l'« art-témoin ${ }^{10}$ ", suggère en creux un portrait du collecteur de voix et une méthode très proche de celle de Jean Hatzfeld à Nyamata, à la fois dans la phase de collecte et dans le travail du montage des témoignages, même si les supports et les langages diffèrent.

9 À partir de cette méthode de l'écoute, inscrite dans une temporalité longue, la trilogie déploie une poétique du témoignage indirect. Le travail de transposition et de composition des voix recueillies manifeste un désir de littérarisation du matériau documentaire. Catherine Coquio et Fleur Kuhn-Kennedy ont souligné la poétisation du témoignage dans la trilogie, dans l'attention esthétique à la langue des rescapés : elles ont montré qu'en maintenant des termes archaïsants et en valorisant les expressions visuelles, comme on le voit bien avec chaque titre issu d'une citation à caractère métaphorique, l'écriture de Jean Hatzfeld se caractérise par une poétique de l'étrangeté dont le but est d'introduire le lecteur dans l'indicible de l'univers génocidaire ${ }^{11}$.

10 Du côté de la composition des livres, s'observe là aussi un travail de littérarisation du matériau, avec une évolution des choix d'un livre à l'autre : chaque livre reflète le désir de trouver une forme capable de s'adapter à la singularité de chaque situation d'entretien. Dans le nu de la vie tient à la fois du récit de voyage, dont la structure inscrit l'écriture en l'espace d'une journée au cours de laquelle le lecteur parcourt les collines de Nyamata en compagnie de l'écrivain, et de la tradition du conte avec le choix d'orchestrer les témoignages dans une scénographie de la rencontre: chaque nouveau témoin qui surgit le long de la route marque le début d'un nouveau chapitre dans lequel le rescapé raconte son histoire. La composition du livre rappelle le dispositif narratif des « hommes-récits » qui caractérise selon Todorov les contes des Mille et une nuits, où chaque rencontre du narrateur avec un nouveau personnage enclenche un nouveau récit enchâssé12 ${ }^{12}$ D'autre part, en maintenant les quatorze récits de ceux qui ont accepté de témoigner, Jean Hatzfeld fait le choix de la sérialité au lieu de la synthèse. Malgré le travail de condensation, la série de témoignages conserve les répétitions d'un récit à l'autre, ce qui rejoint l'ambition éthique du projet : en proposant un récit de vie continu et chronologique, la transcription renforce la singularité de chaque voix ; elle suggère que par le récit le témoin entreprend aussi de reconstituer une identité brisée et de renouer une trajectoire coupée.

11 Dans Une saison de machettes et dans La Stratégie des antilopes, l'écrivain abandonne cette sérialité pour préférer un travail de coupe et de montage sur le modèle cinématographique: la forme s'adapte aux spécificités de nouvelles situations d'entretien. Pour Une saison de machettes, il s'agit cette fois de transmettre une parole collective du point de vue non plus des victimes mais des tueurs, et donc de s'adresser 
«non pas à une suite d'individus mais à un groupe d'individus» (SM, p. 50) : l'enjeu n'est plus de convaincre les rescapés d'élargir le cercle de circulation des témoignages, mais d'explorer les formes prises par la responsabilité collective. L'écriture sérielle du récit de vie laisse place à une écriture chorale thématique : pour faire entrer le lecteur dans la fabrique de l'enquête, l'auteur affirme bien plus sa présence que dans le premier livre. Il propose un discours d'escorte qui remet en perspective les témoignages des bourreaux, entre aveux, mensonges et revirements. Le choix du montage des voix situe La Stratégie des antilopes dans la continuité du deuxième livre: non pas tant ici pour recueillir la parole d'un groupe, mais pour rapprocher dans l'espace du livre des perspectives antagonistes, nécessairement inconciliables, qui sont forcées de cohabiter dans le cadre de la libération et du retour des tueurs. Face à l'échec des gaçaça, ces tribunaux populaires mis en place pour juger les tueurs relâchés, le livre intègre la conflictualité et offre quelque chose comme un exutoire collectif. L'organisation thématique des chapitres permet d'aborder les différents points de vue des rescapés et des bourreaux sur des sujets existentiels (le rapport à la mémoire, aux morts, au pardon), que l'écrivain prend soin d'introduire avant de livrer les extraits de témoignages et de les commenter.

Ces quelques remarques sur la composition montrent aussi à quel point l'évolution de la forme dans la trilogie reflète l'intégration progressive de Jean Hatzfeld à Nyamata, du cloisonnement des voix narratives dans le premier livre au montage du dernier, qui mêle les questions de l'écrivain aux points de vue des témoins. Du journalisme à la littérature, sa mission devient celle d'un passeur de témoignages.

\section{Passeur de témoignages : enjeux de la réception}

Dans la trilogie, le livre apparait comme un horizon indépassable, ce dont témoigne le titre de l'intervention de Jean Hatzfeld aux Assises du roman : «Les bouquins d'abord ». Le livre est à la fois un objet de désir vers lequel tend la collecte, venant lui donner un sens ; un objet de négociation avec les participants, rescapés et bourreaux ; tandis qu'en retour il offre un instrument de légitimation de la présence et du travail de l'écrivain, comme on le voit dans La Stratégie des antilopes où l'écrivain revient sur la réception du premier livre auprès des rescapés :

Non seulement le Français n'a pas trahi, mais en plus tout le monde a reconnu avoir beaucoup appris sur le génocide à la lecture du livre, même les gens qui y ont participé, car ils ont lu et retrouvé dans leurs propres récits des idées ou des impressions formulées, qu'ils gardaient jusque-là implicites ou secrètes, souvent confuses, parce que trop douloureuses ou, croyaient-elles, inintelligibles, invraisemblables, suspectes pour autrui. (SA, p. 208)

14 La réussite se traduit ici par le talent de médiation de l'écrivain, capable de révéler l'autre à lui-même, et au-delà de permettre à la communauté des rescapés de se reconnaître et d'advenir à elle-même. Le livre devient ainsi une étape essentielle dans le processus de familiarisation et de confiance entre l'écrivain et les témoins; d'où la mise en place après chaque livre d'un protocole de contre-visite intégré au livre suivant, afin d'éprouver sa pertinence et ses effets pragmatiques. Jean Hatzfeld détaille ainsi dans La Stratégie des antilopes la réaction des rescapés à la lecture des livres, et l'évolution du rapport de chacun au témoignage : alors qu'Odette et Édith en viennent à refuser de parler, certains consolident leur statut social de témoin, à l'instar de Cassius : «[...] il admet qu'il consacre toute son énergie au souvenir du génocide, sur 
quoi il a beaucoup écouté et réfléchi, et qu'il a appris à raconter en phrases peaufinées, au fil des sollicitations dont il est l'objet, avec sincérité » (SA, p. 195).

Mais si la réception locale est essentielle à la poursuite du projet, ce dernier vise avant tout un public européen : dès l'introduction de Dans le nu de la vie, l'auteur précise que son but n'est pas d'ajouter une nouvelle enquête aux enquêtes préexistantes, mais «uniquement de faire lire ces étonnants récits de rescapés» (DNV, p.9) afin de contribuer à élargir la mémoire du génocide de l'échelle locale à l'échelle internationale. Il faut souligner à cet égard la très grande pédagogie de la trilogie : d'un point de vue visuel, on pense aux cartes du Rwanda et du district de Nyamata qui accompagnent chaque livre, tandis que l'appareil paratextuel offre pour chaque volume un glossaire des termes non-traduits du kinyarwanda, qu'ils relèvent du vocabulaire du génocide, de la culture ou de la géographie rwandaises, ainsi qu'une chronologie augmentée pour chaque livre. Enfin, les références au génocide juif jouent un rôle évident dans le travail de transmission de la mémoire du génocide tutsi. La mise en parallèle des paroles des rescapés avec les réflexions de Primo Levi ou de Charlotte Delbo assurent une fonction de reconnaissance aux yeux du lecteur européen, tressant une continuité entre témoins de la Shoah et témoins tutsis. Les livres de Jean Hatzfeld ont joué un rôle important dans la réception française du génocide au tournant des années $2000^{13}$. Le succès des recueils de témoignages et leur force d'incarnation ont de plus eu des effets pragmatiques très significatifs, puisque l'auteur mentionne dans un entretien le cas de nombreux lecteurs partis sur ses traces au Rwanda dans l'espoir de rencontrer les témoins et de les interroger à leur tour. Le livre suscite ainsi de nouvelles enquêtes, il provoque de nouveaux départs.

Pour conclure cette question de la réception, on peut toutefois nuancer l'affirmation d'une rupture nette avec l'expérience du journalisme évoquée plus haut. Du point de vue de la programmation de la réception, la notoriété de Jean Hatzfeld en tant que grand reporter de guerre n'a pu que contribuer au succès de la trilogie : les nombreuses références à son expérience en Yougoslavie servent de caution aux analyses sur la singularité de l'expérience génocidaire. Les quatrièmes de couverture le présentent à la fois comme écrivain et journaliste : le second métier sert ici de gage d'autorité et vient apporter, dans le cadre d'une littérature documentaire, une légitimité au projet de l'écrivain. L'affirmation d'une rupture radicale entre le reporter et l'écrivain relève donc aussi d'une posture : c'est une stratégie de légitimation pour se faire reconnaître dans le champ littéraire en tant qu'écrivain et non comme journaliste. Il y aurait d'ailleurs matière à étudier comment Dans le nu de la vie prolonge en réalité les portraits de témoins parus dans Libération en 1998.

\section{Les politiques du reportage}

L'exemple de Jean Hatzfeld reflète la complexité des rapports entre politique et littérature pour de nombreux écrivains contemporains. L'article de Bruno Blanckeman sur «l'écrivain impliqué » faisait l'hypothèse d'un infléchissement de la responsabilité littéraire au tournant des années 2000, de l'engagement à l'implication dans la cité : il s'agit moins, pour les écrivains contemporains, de faire entendre une parole engagée que de s'impliquer dans des sujets sensibles, qu'ils abordent en refusant de résoudre l'opacité et la complexité des situations, en cherchant des formes d'intervention moins surplombantes, plus inquiètes ${ }^{14}$. Dans son travail sur le génocide tutsi, Jean Hatzfeld se 
distingue d'abord par son retrait du débat politique. Dès les années 2000, les relations entre la France et le Rwanda sont très tendues: en 2004, Paul Kagamé accuse le gouvernement français de complicité avec les responsables du génocide; encore en 2017, Patrick de Saint-Exupéry publie dans XXI un article sur le dévoilement de sources qui prouvent la compromission d'Hubert Védrine avec le gouvernement hutu en avril 1994'15. En tant qu'écrivain français enquêtant sur une commune rwandaise meurtrie par le génocide, jouissant d'un certain capital symbolique à la fois dans le champ journalistique et dans le champ littéraire avec la parution des premiers livres, on pourrait attendre que Jean Hatzfeld s'exprime dans le cadre des polémiques qui agitent le débat public. Mais sur ce sujet, il ne se prononce jamais directement, ni dans les livres, ni dans ses interventions médiatiques, refusant toute prise de position sur la responsabilité de la France. La chronologie à la fin de chaque livre précise seulement que l'opération Turquoise est " ambiguë » et qu'elle « suscite encore des polémiques ${ }^{16}$ ». Sa position consiste à relayer dans les livres l'amertume des rescapés face à l'abandon dont ils ont fait l'objet en 1994. Il explique qu'une part de leur suspicion à son égard est liée à cette méfiance envers les Blancs. On trouve ainsi dans la bouche d'Innocent une charge directe contre le gouvernement français, avec la scène récurrente dans la trilogie de l'évacuation des ressortissants étrangers avant les premiers massacres :

Par ailleurs, des Français savaient que le génocide se préparait, puisqu'ils recommandaient notre armée. Soi-disant, ils n'y croyaient pas ; pourtant beaucoup de Blancs connaissaient le programme et le caractère d'Habyarimana [...]. Un jour, à Nyamata, des blindés blancs sont venus afin de récupérer les pères blancs. Dans la grand-rue, les interahamwe ont cru qu'ils venaient pour les punir et ils se sont enfuis en se criant les uns les autres que les Blancs allaient les tuer. Les chars n'ont même pas marqué une petite pause Primus pour rigoler du quiproquo. Et, quelques semaines plus tard, les Blancs ont envoyé des photographes professionnels pour montrer au monde comment on était massacrés. Alors, vous pouvez comprendre que dans le cœur des rescapés il s'est glissé un sentiment d'abandon qui ne se dissipera jamais. Mais, je ne veux pas vous fâcher avec ça. (DNV, p. 107)

Derrière la délicatesse d'Innocent, la prise à parti finale rappelle à l'écrivain qu'il appartient à un pays complice, dont le récit souligne régulièrement la responsabilité. C'est une façon aussi de situer l'écrivain, de le remettre à sa place, en formulant une mise en garde contre une approche trop compassionnelle ou empathique. À l'engagement frontal se substitue donc un parti pris qui consiste à relayer les témoignages et avis de chacun, plutôt qu'à s'exprimer en son nom propre; à expérimenter des manières de rapporter différents points de vue sans s'arroger le magistère du porte-parole, plutôt que d'entreprendre une possible investigation politique.

19 L'intervention politique de l'écrivain se fait donc sur un mode plus indirect, plus ténu aussi ; mais ce choix du passeur permet peut-être d'aborder des questions politiques plus fondamentales. Ainsi, davantage que les deux premiers livres, La Stratégie des antilopes procède directement de la question suivante: comment peut-on vivre ensemble après le génocide ? La collecte de voix permet cette fois d'aller voir ce qui se cache derrière la façade officielle de la réconciliation nationale, du "Plan Pardon », pour en montrer les lézardes. L'observation ethnographique devient plus aiguë que dans les premiers volumes dans la mesure où l'écrivain en vient peu à peu à distinguer différentes modalités de la parole selon les lieux où elle s'exerce : l'échec des gaçaças où le témoignage des Tutsis comme des Hutus est placé sous contrôle ; le rôle des cabarets où les points de vue s'expriment plus librement mais avec un risque de violence latent; 
le cadre privé où les demandes de pardon restent souvent lettre morte. Jean Hatzfeld pointe ainsi les freins à l'émergence d'un débat public, une aporie qu'exprime bien Innocent lorsqu'il regrette qu'«il n'existe aucune occasion, pour les rescapés, d'exprimer en public leurs vrais sentiments intimes » (SA, p. 111). Dès lors, dans ce contexte de cloisonnement entre l'espace privé des confidences et l'espace public d'une parole sous contrôle, l'écrivain occupe une place singulière. Non seulement la parole qui lui est confiée n'appartient plus tout à fait à la sphère intime, puisque le contrat de l'entretien repose sur la publication du témoignage, à entendre au double sens du terme: publier et rendre public, mais le livre apparait dans cette configuration spécifique comme un espace d'expression, un dispositif alternatif aux cadres de la parole fixés par le pouvoir. Cette situation en marge des enjeux politiques immédiats permet aux rescapés de redire à quel point l'absence de réparation symbolique et matérielle fait obstacle à une réconciliation réelle. D'autre part, c'est parce que le témoignage n'a pas la même portée judiciaire dans le tribunal et dans le livre, où les conséquences directes sont restreintes, qu'Hatzfeld parvient aussi à recueillir les témoignages des tueurs et à faire entendre dans l'espace du livre l'opposition de points de vue inconciliables, tout en ménageant des espaces de singularité au sein de chaque groupe. L'exposition des dissensus et la confrontation des points de tension permettent d'interroger les obstacles et les éventuelles manières de refaire communauté.

Revenons à la citation de départ: ainsi, l'opposition frontale entre journalisme et littérature, entre "ligne droite" et "ligne sinueuse", occulte les nombreuses passerelles qui relient la pratique du reporter au travail de l'écrivain à Nyamata. Mais ce parti pris de l'oblique révèle les réajustements d'une méthode d'enquête qui passe par le choix du témoignage indirect. La façon de transposer les récits, l'adaptation du protocole et de la forme des livres à de nouvelles situations, la quête d'une juste distance, impliquent autant une politique qu'une éthique du reportage, où s'affirme, en même temps que le refus de se faire porte-parole, une réelle implication de passeur. Construite depuis deux décennies, cette figure de passeur ne cesse d'être réinvestie dans les apparitions médiatiques de l'écrivain: en public et dans ses discours, Jean Hatzfeld continue à porter à un public occidental cette parole collectée auprès des rescapés, citant volontiers telle réflexion d'Innocent Rwililiza ou telle remarque de Sylvie Umubyeyi. Ce que montre la politique relativement diffuse et ténue du reportage mise en œuvre par Jean Hatzfeld, c'est donc à la fois la recherche d'une forme responsable et l'adoption d'une posture de porte-voix plutôt que de porte-parole.

\section{NOTES}

1. Les références renvoient aux éditions suivantes: J. Hatzfeld, Dans le nu de la vie : récits des marais rwandais, Paris, Seuil, coll. « Points », 2000 (dorénavant DNV); Une saison de machettes, Paris, Seuil, 
coll. «Points », 2003 (dorénavant SM) ; La Stratégie des antilopes, Paris, coll. « Points ", 2007 (dorénavant SA).

2. Selon les travaux de l'historienne A. Wieviorka, L'Ère du témoin, Paris, Plon, 1998.

3. J. Hatzfeld, "Les bouquins d'abord", Assises internationales du roman 2012, Penser pour mieux rêver, Paris, Christian Bourgois, 2012, p. 165-167, p. 167.

4. Voir C. Delporte, C. Blandin et F. Robinet, Histoire de la presse en France, $\mathrm{XX}^{e}-\mathrm{XXI}^{e}$ siècles, Paris, Armand Colin, coll. «U $», 2016$.

5. J. Hatzfeld, «La littérature est devenue un refuge », entretien avec Jean Birnbaum, 6 mai 2010, en ligne sur Le Monde des livres: <http://www.lemonde.fr/livres/article/2010/05/06/jeanhatzfeld-la-litterature-est-devenue-un-refuge_1347329_3260.html> [consulté le 12 mai 2020].

6. Contraction en anglais de magazine et de book, le mook désigne un type de publication papier à mi-chemin entre le magazine et le livre. Ces nouvelles revues, incarnées en France par Feuilleton, XXI ou encore Usbek \& Rika, privilégient le reportage au long cours.

7. La revue XXI est fondée en 2008 par Patrick de Saint-Exupéry.

8. Voir A. Caillet, Dispositifs critiques: le documentaire, du cinéma aux arts visuels, Rennes, Presses Universitaires de Rennes, coll. « Arts contemporains », 2014, p. 60-61.

9. J. Hatzfeld, « Pourquoi lui ?», dans J. Simont (dir.), Claude Lanzmann: un voyant dans le siècle, Paris, Gallimard, 2017, p. 145-151, p. 147.

10. Voir S. Felman, «À l'âge du témoignage : Shoah de Claude Lanzmann », dans M. Deguy (dir.), Au sujet de Shoah, Paris, Belin, 1990, p.55-145. Pour une analyse de l'écho du commentaire de Shoshana Felman dans la littérature de témoignage, voir C. Coquio, La Littérature en suspens. Écritures de la Shoah : le témoignage et les cuvres, Paris, L'Arachnéen, 2015, p. 67-88.

11. Voir C. Coquio, Rwanda: le réel et les récits, Paris, Belin, 2004, p. 169-177 ; F. Kuhn-Kennedy, " "À voix haute ou silencieuse": Dans le nu de la vie de Jean Hatzfeld et les médiations du témoignage ", Cahiers ERTA, n 8, « Narrations », 2015, p. 89-100.

12. T. Todorov, Poétique de la prose [1971], «Les hommes-récits », Paris, Seuil, coll. « Points", 1978, p. 33-46.

13. Voir C. Coquio, Rwanda : le réel et les récits, ouvr. cité, p. 97.

14. B. Blanckeman, "L'écrivain impliqué : écrire (dans) la cité », dans Narrations d'un nouveau siècle : romans et récits français (2001-2010), colloque de Cerisy, Paris, Presses Sorbonne Nouvelle, 2012, p. 71-81.

15. P. de Saint-Exupéry, « Réarmez-les ! », XXI, n 39, « Nos crimes en Afrique », été 2017, p. 56-65. 16. Fin juin 1994, la France est chargée par l'ONU de mettre en place une zone tampon à la frontière congolaise, accusée d'avoir facilité la fuite des responsables du génocide.

\section{RÉSUMÉS}

Cet article étudie la manière dont se négocient les rapports entre reportage et littérature dans la trilogie rwandaise de Jean Hatzfeld (Dans le nu de la vie; Une saison de machettes; La Stratégie des antilopes). Dans ces recueils de témoignages, l'écrivain revendique une césure nette avec sa pratique de grand reporter, opposant dans La Stratégie des antilopes la «ligne droite» du journalisme à la « ligne sinueuse » de la littérature. Il s'agit ici d'interroger cet infléchissement selon trois axes : revenir sur l'invention dans la trilogie d'une éthique de l'écoute et d'une 
poétique du témoignage indirect; montrer comment la réception complexifie ce partage entre reportage et littérature ; interroger les liens entre politique et reportage au fil des livres.

This paper discusses the relationships between reportage and literature in Jean Hatzfeld's Rwandan trilogy (Dans le nu de la vie, Une saison de machettes and La Stratégie des antilopes). By collecting testimonies, the writer claims a clear break with his former practice as reporter, setting in La Stratégie des antilopes the "direct line" of journalism against the "sinuous line" of literature. This paper aims to question this evolution according to three aspects: how Jean Hatzfeld invents an ethic based on listening and a poetic of indirect testimony throughout the trilogy; how reception complexifies the gap between reportage and literature; how the books redefine the relationships between politics and literary reportage.

\section{INDEX}

Mots-clés : témoignage, entretien, journalisme, polyphonie, terrain

Keywords : testimony, interview, journalism, polyphony, fieldwork

\section{AUTEUR}

\section{MAUD LECACHEUR}

Maud Lecacheur est doctorante en littérature française contemporaine à l'ENS de Lyon. Sa thèse, « Postures d'écrivains publics : recueillir la parole d'autrui de Georges Perec à Olivia Rosenthal », porte sur les enjeux esthétiques, éthiques et politiques des collectes de voix contemporaines. Elle a publié un article sur ces dispositifs dans Fixxion, ainsi que plusieurs articles sur Olivia Rosenthal et Jean-Paul Goux. 